Wright State University

CORE Scholar

Kno.e.sis Publications

The Ohio Center of Excellence in Knowledge-

Enabled Computing (Kno.e.sis)

$9-2013$

\title{
A Statistical and Schema Independent Approach to Identify Equivalent Properties on Linked Data
}

\author{
Kalpa Gunaratna \\ Wright State University - Main Campus, gunaratna.2@wright.edu \\ Krishnaprasad Thirunarayan \\ Wright State University - Main Campus, t.k.prasad@wright.edu \\ Prateek Jain \\ Wright State University - Main Campus \\ Amit P. Sheth \\ Wright State University - Main Campus, amit@sc.edu \\ Sanjaya Wijeratne \\ Wright State University - Main Campus, wijeratne.2@wright.edu
}

Follow this and additional works at: https://corescholar.libraries.wright.edu/knoesis

Part of the Bioinformatics Commons, Communication Technology and New Media Commons, Databases and Information Systems Commons, OS and Networks Commons, and the Science and Technology Studies Commons

\section{Repository Citation}

Gunaratna, K., Thirunarayan, K., Jain, P., Sheth, A. P., \& Wijeratne, S. (2013). A Statistical and Schema Independent Approach to Identify Equivalent Properties on Linked Data. .

https://corescholar.libraries.wright.edu/knoesis/565

This Presentation is brought to you for free and open access by the The Ohio Center of Excellence in KnowledgeEnabled Computing (Kno.e.sis) at CORE Scholar. It has been accepted for inclusion in Kno.e.sis Publications by an authorized administrator of CORE Scholar. For more information, please contact library-corescholar@wright.edu. 


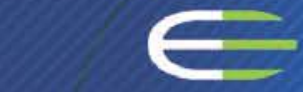

\section{A Statistical and Schema Independent Approach to Identify Equivalent Properties on Linked Data}

Kalpa Gunaratna ${ }^{\dagger}$, Krishnaprasad Thirunarayan $^{\dagger}$, Prateek Jain ${ }^{\ddagger}$, Amit Sheth $^{\dagger}$, and Sanjaya Wijeratne ${ }^{\dagger}$

\{kalpa,tkprasad,amit,sanjaya\}@knoesis.org, jainpr@us.ibm.com

${ }^{\dagger}$ Kno.e.sis Center

Wright State University

Dayton OH, USA
${ }^{\ddagger}$ IBM T J Watson Research Center

Yorktown Heights

New York NY, USA 


\section{Why we need property alignment and it is so important?}

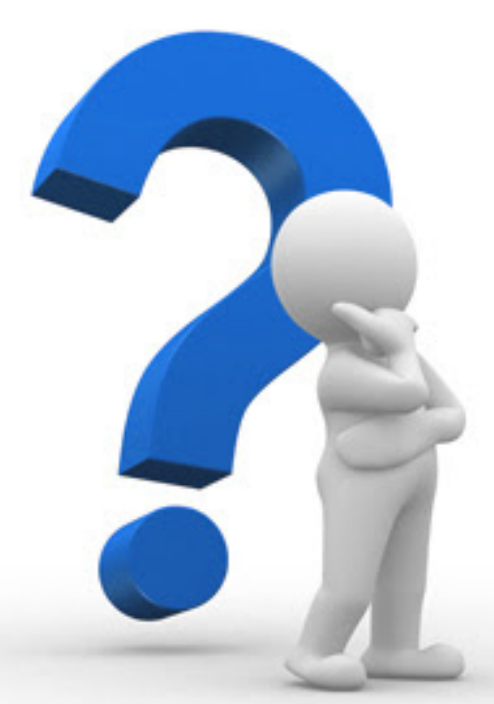




\section{Roadmap}

\section{$\square$ Background}

\section{$\square$ Statistical Equivalence of properties}

$\square$ Evaluation

$\square$ Discussion, interesting facts, and future directions

$\square$ Conclusion 
$\square$ Existing techniques for property alignment fall into three categories.

I. Syntactic/dictionary based

- $\quad$ Uses string manipulation techniques, external dictionaries and lexical databases like WordNet.

II. Schema dependent

- Uses schema information such as, domain and range, definitions.

III. Schema independent

- Uses instance level information for the alignment.

$\square$ Our approach falls under schema independent. 
$\square$ Properties capture meaning of triples and hence they are complex in nature.

$\square$ Syntactic or dictionary based approaches analyze property names for equivalence. But in LOD, name heterogeneities exist.

$\square$ Therefore, syntactic or dictionary based approaches have limited coverage in property alignment.

$\square$ Schema dependent approaches including processing domain and range, class level tags do not capture semantics of properties well. 


\section{Roadmap}

Background

$\square$ Statistical Equivalence of properties

DEvaluation

$\square$ Discussion, interesting facts, and future directions

aConclusion 


\section{Statistical Equivalence of properties}

$\square$ Statistical Equivalence is based on analyzing owl:equivalentProperty.

$\square$ owl:equivalentProperty - properties that have same property extensions.

\section{Example 1:}

Property $P$ is defined by the triples, $\{a P b, c P d, e P f\}$

Property $Q$ is defined by the triples, $\{a Q b, c Q d, e Q f\}$

$P$ and $Q$ are owl:equivalentProperty, because they have the same extension, $\{\{a, b\},\{c, d\},\{e, f\}\}$

\section{Example 2:}

Property $P$ is defined by the triples, $\{a P b, c P d, e P f\}$

Property $Q$ is defined by the triples, $\{a Q b, c Q d, e Q h\}$

Then, $P$ and $Q$ are not owl:equivalentProperty, because their extensions are not the same. But they provide statistical evidence in support of equivalence. 


\section{Intuition}

$\square$ Higher rate of subject-object matches in extensions leads to equivalent properties. In practice, it is hard to have exact same extensions for matching properties. Because,

- Datasets are incomplete.

- Same instance may be modelled differently in different datasets.

$\square$ Therefore, we analyze the property extensions to identify equivalent properties between datasets.

$\square$ We define the following notions. Let the statement below be true for all the definitions.

$S_{1} P_{1} O_{1}$ and $S_{2} P_{2} O_{2}$ be two triples in Dataset $D_{1}$ and $D_{2}$ respectively. 


\section{Definition 1: Candidate Match}

The two properties $P_{1}$ and $P_{2}$ are a candidate match iff $\mathrm{S}_{1} \stackrel{E C R *}{\longleftrightarrow} \mathrm{S}_{2}$ and $\mathrm{O}_{1} \stackrel{E C R *}{\longleftrightarrow} \mathrm{O}_{2}$.

We say two instances are connected by an $E C R^{*}$ link if there is a link path between the instances using ECR links ( ${ }^{*}$ is the Kleene star notation). ECR links are Entity Co-reference Relationships such as those formalized using owl:sameAs and skos:exactMatch.

\section{Example}

The two datasets DBpedia(d) and Freebase(f)

$d:$ Arthur Purdy Stout

d:place of birth $\quad d:$ New York City

f:Arthur Purdy Stout

f:place of death $\quad f:$ New York City

The above is a candidate match, but not equivalent, because intensions are different (coincidental match).

$\square$ We need further analysis to decide on equivalence. 
Match Count $\mu\left(P_{1}, P_{2}\right)-$ Number of triple pairs for $P_{1}$ and $P_{2}$ that participate in candidate matches.

$$
\mu\left(P_{1}, P_{2}\right)=\left|\left\{S_{1} P_{1} O_{1} \in D_{1} \mid \exists S_{2} P_{2} O_{2} \in D_{2} \wedge S_{1} \stackrel{E C R *}{\longrightarrow} S_{2} \wedge O_{1} \stackrel{E C R *}{\longrightarrow} O_{2}\right\}\right|
$$

Co-appearance Count $\lambda\left(P_{1}, P_{2}\right)-$ Number of triple pairs for $P_{1}$ and $P_{2}$ that have matching subjects.

$$
\lambda\left(P_{1}, P_{2}\right)=\left|\left\{S_{1} P_{1} O_{1} \in D_{1} \mid \exists S_{2} P_{2} O_{2} \in D_{2} \wedge S_{1} \stackrel{E C R *}{\longrightarrow} S_{2}\right\}\right|
$$

\section{Definition 2: Statistically Equivalent Properties}

The pair of properties $P_{1}$ and $P_{2}$ are statistically equivalent to degree $(\alpha, \mathrm{k})$ iff,

$$
F=\frac{\mu\left(P_{1}, P_{2}\right)}{\lambda\left(P_{1}, P_{2}\right)} \geq \alpha,
$$

Where, $\mu\left(P_{1}, P_{2}\right) \geq \mathrm{k}$, and $0<\alpha \leq 1, \mathrm{k}>1$ 


\section{Candidate Matching Algorithm Process}

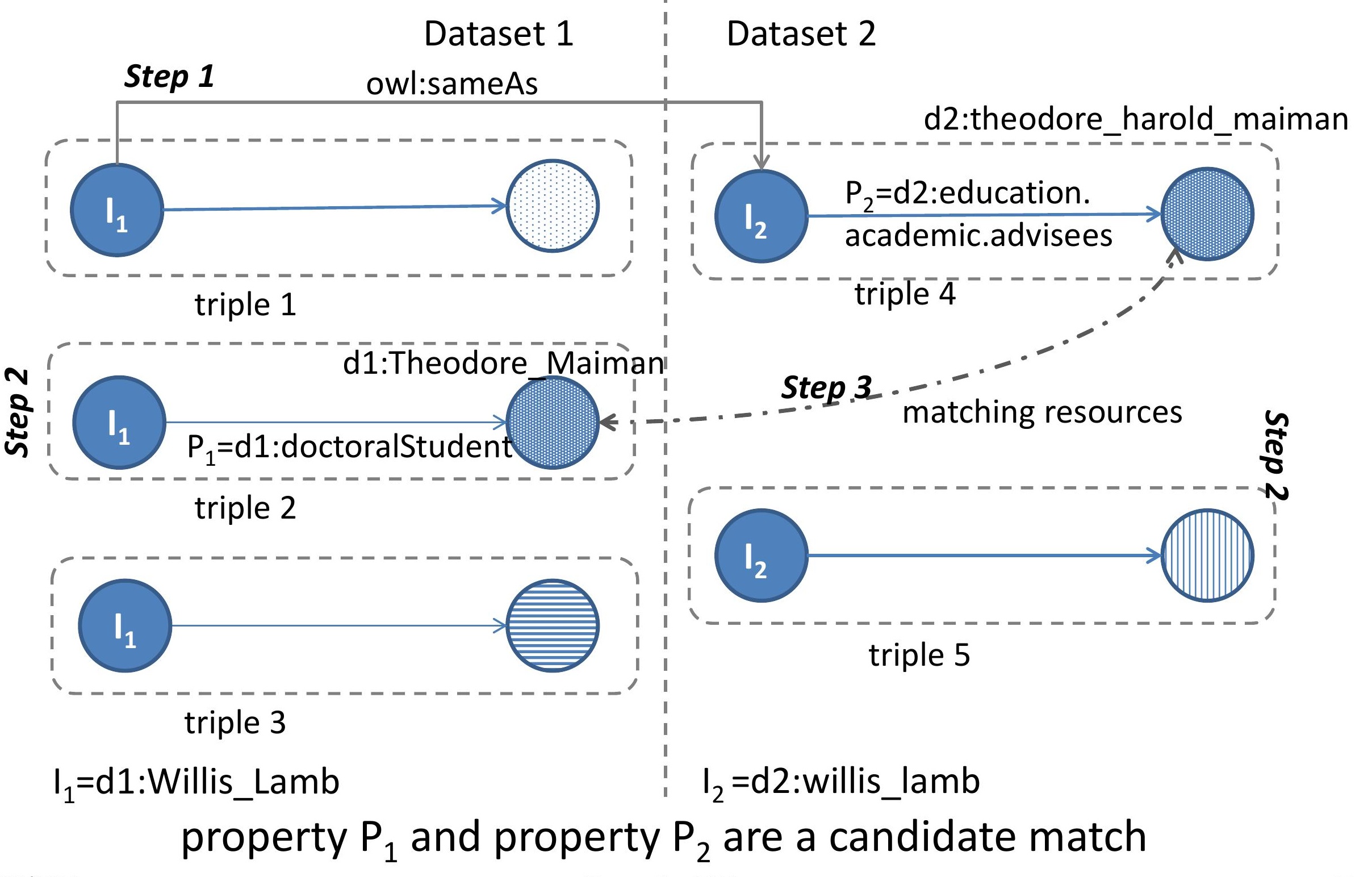




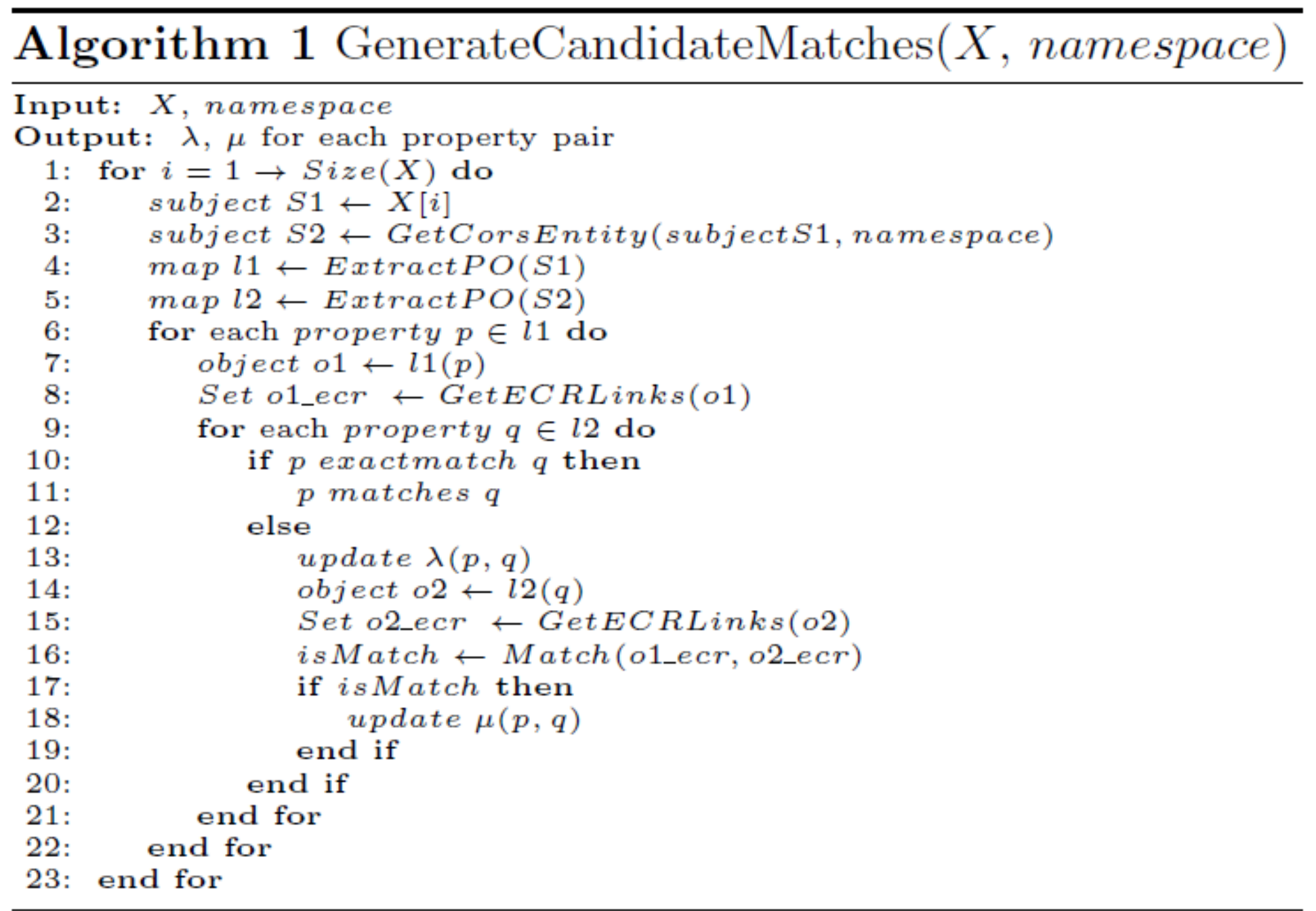

\section{Complexity:}

If the average number of properties for an entity is $x$ and for each property, average number of objects is $j$. For $n$ subjects, it requires $n^{*} \mathbf{j}^{2 *} \mathbf{x}^{\mathbf{2}}+\mathbf{2 n}$ comparisons. Since $n>j$, $n>x$, and $x$ and $j$ are independent of $n, O(n)$. 


\section{Example:}

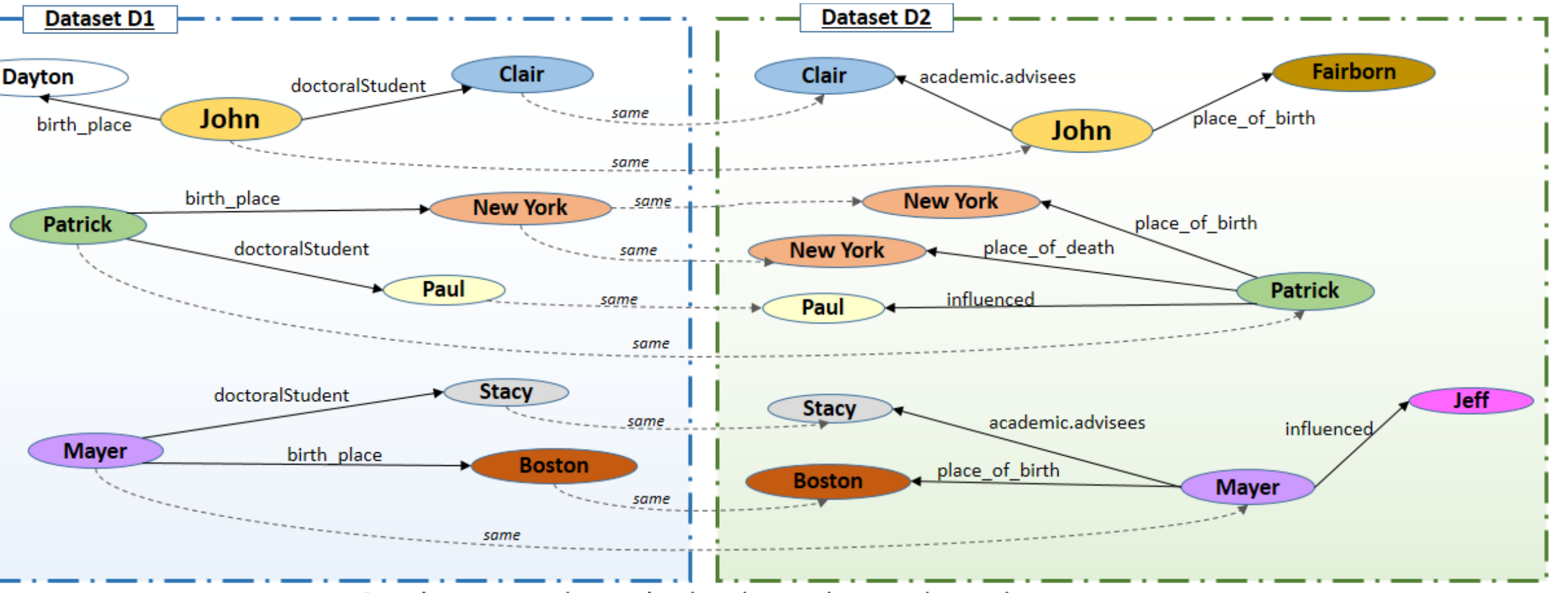

Same instances are shown using dotted arrows between the two datasets.

\section{Generated Candidate Matching Property Lists - Matches selected are in Boldface}

[D1:doctoralStudent] [ [D2:academic.advisees, 2:2], [D2:influenced, 1:2] ]

$\longleftarrow$ matching list for doctoralStudent

[D1:birth_place]

[ [D2:place_of_birth, 2:3], [D2:place_of_death, 1:1]]

matching list for birth_place

\begin{tabular}{|l|c|c|}
\hline \multicolumn{1}{|c|}{ Property Pair (matched) } & MatchCount & Co-appearanceCount \\
\hline [D1:doctoralStudent, D2:academic.advisees] & 2 & 2 \\
\hline [D1:birth_place, D2:place_of_birth] & 2 & 3 \\
\hline
\end{tabular}

Selection using $\alpha=0.5$ and $k=2$ 


\section{Parallel computation (Map-Reduce implementation)}

- Generating candidate matches can be done for each instance independently. Hence, we implemented the algorithm in Hadoop 1.0.3 framework.

$\square$ Generating candidate matches for instances is distributed among mappers and each mapper outputs $\mu$ and $\lambda$ to the reducer for property pairs.

- Map Phase

- Let the number of subject instances in dataset $D_{1}$ be $X$ and namespace of dataset $\mathrm{D}_{2}$ be ns. For each subject $i \in X$, start a mapper job for GenerateCandidateMatches $(i, n s)$.

- Each mapper outputs (key,value) pairs as $(p: q, \mu(p, q): \lambda(p, q))$. $p \in D_{1}$ and $q \in D_{2}$.

$\square$ The reducer collects all $\mu$ and $\lambda$ values and aggregate them for final analysis.

- Reduce phase

- Collects output from mappers and aggregates $\mu(p, q)$ and $\lambda(p, q)$ for each key $p: q$.

$\square$ The map reduce version on a 14 node cluster was able to achieve a speed up of $833 \%$ compared to the desktop version. 


\section{Roadmap}

Background

Q Statistical Equivalence of properties

$\square$ Evaluation

$\square$ Discussion, interesting facts, and future directions

a Conclusion 
$\square$ Objectives of the evaluation

- Show the effectiveness of the approach in linked datasets

- Compare with existing aligning techniques

$\square$ We selected 5000 instance samples from DBpedia, Freebase, LinkedMDB, DBLP L3S, and DBLP RKB Explorer datasets.

$\square$ These datasets have,

- Complete data for instances in different viewpoints

- Many inter-links

- Complex properties 
$\square$ Experiment details

$-\alpha=0.5$ for all experiments (works for LOD) except DBpedia and Freebase movie alignment where it was 0.7.

DBpedia-Freebase (Person)

$-k$ was Softw Linker

$-k$ can

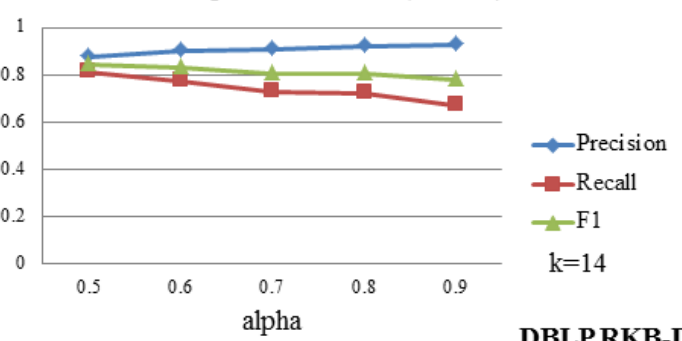

DBLP RKB-DBLP L3S (Article)
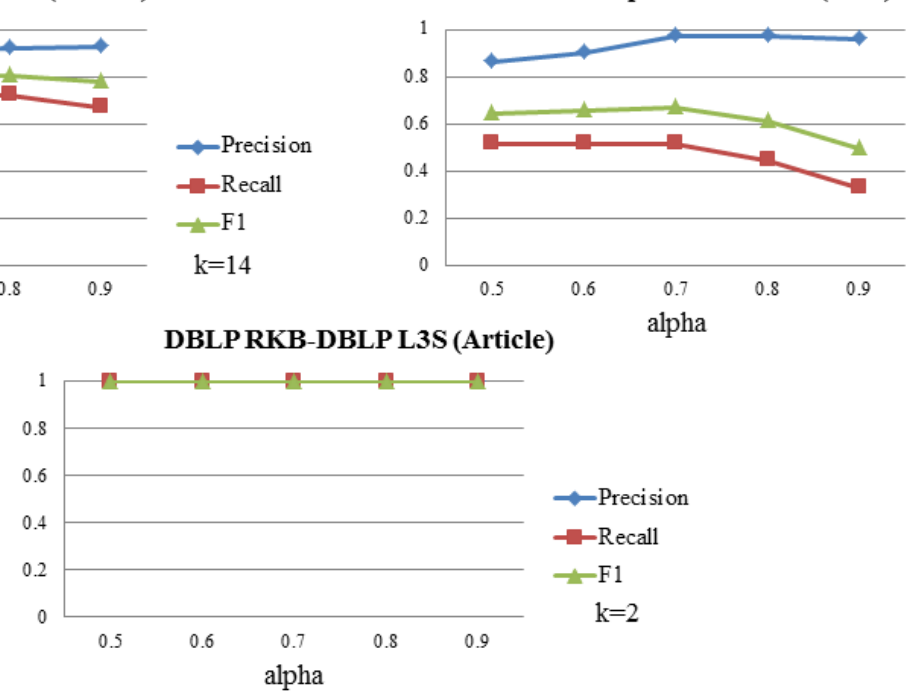

DBpedia-LinkedMDB (Film)
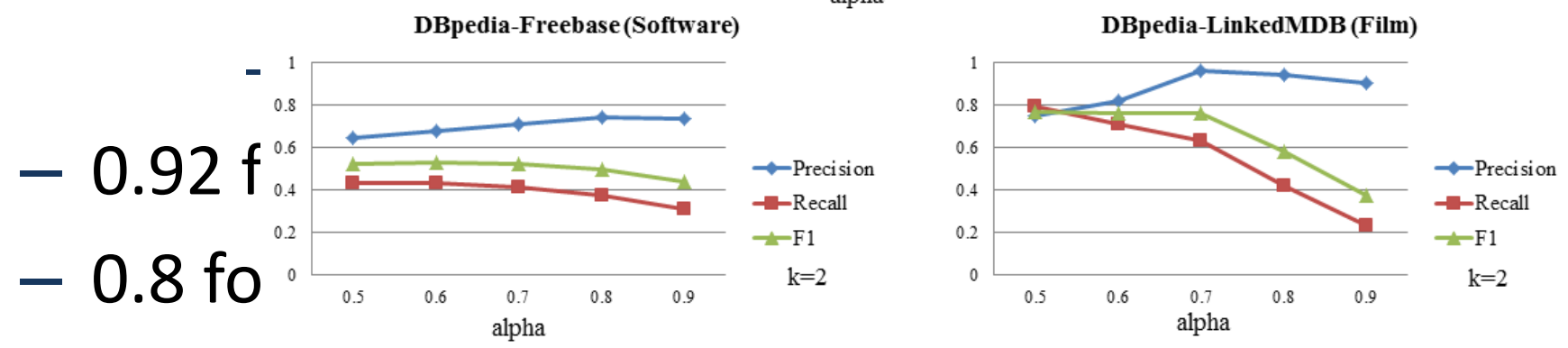

son, Film and

$\rightarrow \rightarrow$ pesisian etween

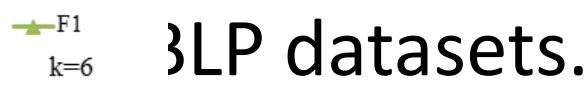

rs not identified by 


\section{$\underline{\text { Alignment results }}$}

\begin{tabular}{|c|c|c|c|c|c|c|c|}
\hline & $\begin{array}{l}\text { Measure } \\
\text { type }\end{array}$ & $\begin{array}{l}\text { DBpedia - } \\
\text { Freebase } \\
\text { (Person) }\end{array}$ & $\begin{array}{l}\text { DBpedia - } \\
\text { Freebase } \\
\text { (Film) }\end{array}$ & $\begin{array}{l}\text { DBpedia - } \\
\text { Freebase } \\
\text { (Software) }\end{array}$ & $\begin{array}{l}\text { DBpedia - } \\
\text { LinkedMDB } \\
\text { (Film) }\end{array}$ & $\begin{array}{l}\text { DBLP_RKB - } \\
\text { DBLP_L3S } \\
\text { (Article) }\end{array}$ & Average \\
\hline \multirow{3}{*}{$\begin{array}{l}\text { Extension } \\
\text { Based } \\
\text { Algorithm }\end{array}$} & Precision & 0.8758 & 0.9737 & 0.6478 & 0.7560 & 1.0000 & 0.8427 \\
\hline & Recall & $0.8089 *$ & 0.5138 & 0.4339 & 0.8157 & 1.0000 & 0.7145 \\
\hline & $\mathrm{F}$ measure & $0.8410 *$ & 0.6727 & 0.5197 & 0.7848 & 1.0000 & 0.7656 \\
\hline \multirow{3}{*}{$\begin{array}{l}\text { WordNet } \\
\text { Similarity }\end{array}$} & Precision & 0.5200 & 0.8620 & 0.7619 & 0.8823 & 1.0000 & 0.8052 \\
\hline & Recall & $0.4140 *$ & 0.3472 & 0.3018 & 0.3947 & 0.3333 & 0.3582 \\
\hline & F measure & $0.4609 *$ & 0.4950 & 0.4324 & 0.5454 & 0.5000 & 0.4867 \\
\hline \multirow{3}{*}{$\begin{array}{l}\text { Dice } \\
\text { Similarity }\end{array}$} & Precision & 0.8064 & 0.9666 & 0.7659 & 1.0000 & 0.0000 & 0.7078 \\
\hline & Recall & $0.4777^{*}$ & 0.4027 & 0.3396 & 0.3421 & 0.0000 & 0.3124 \\
\hline & F measure & $0.6000^{*}$ & 0.5686 & 0.4705 & 0.5098 & 0.0000 & 0.4298 \\
\hline \multirow{3}{*}{$\begin{array}{l}\text { Jaro } \\
\text { Similarity }\end{array}$} & Precision & 0.6774 & 0.8809 & 0.7755 & 0.9411 & 0.0000 & 0.6550 \\
\hline & Recall & $0.5350 *$ & 0.5138 & 0.3584 & 0.4210 & 0.0000 & 0.3656 \\
\hline & F measure & $0.5978 *$ & 0.6491 & 0.4903 & 0.5818 & 0.0000 & 0.4638 \\
\hline
\end{tabular}

* Marks estimated values for experiment 1 because of very large comparisons to check manually. Boldface marks highest result for each experiment. 


\section{$\square$ Example identifications}

\begin{tabular}{l|l|l}
$\begin{array}{l}\text { Property pair } \\
\text { types }\end{array}$ & Dataset 1 (DBpedia) & Dataset 2 (Freebase) \\
\hline $\begin{array}{l}\text { Simple string } \\
\text { similarity matches }\end{array}$ & db:nationality & fb:nationality \\
\cline { 2 - 3 } $\begin{array}{l}\text { Synonymous } \\
\text { matches }\end{array}$ & db:religion & fb:religion \\
\hline Complex matches & db:battles & fb:profession \\
\hline & db:screenplay & fb:participated_in_conflicts \\
\hline & db:doctoralStudent & fb:written_by \\
\hline
\end{tabular}

WordNet similarity failed to identify any of these 


\section{Roadmap}

Background

DStatistical Equivalence of properties

DEvaluation

$\square$ Discussion, interesting facts, and future directions

aConclusion 
$\square$ Our experiment covered multi-domain to multi-domain, multidomain to specific domain and specific-domain to specific-domain dataset property alignment.

$\square$ In every experiment, the extension based algorithm outperformed others ( $\mathrm{F}$ measure). $\mathrm{F}$ measure gain is in the range of $\mathbf{5 7 \%}$ to $\mathbf{7 8 \%}$.

$\square$ Some properties that are identified are intentionally different, e.g., $d b$ :distributor vs $f b$ :production_companies.

- This is because many companies produce and also distribute their films.

$\square$ Some identified pairs are incorrect due to errors in data modeling.

- For example, $d b$ :issue and fb:children.

$\square$ owl:sameAs linking issues in LOD (not linking exact same thing), e.g., linking London and Greater London.

- We believe few misused links wont affect the algorithm as it decides on a match after analyzing many matches for a pair. 
$\square$ Less number of interlinks.

- Evolve over time.

- Look for possible other types of ECR links (i.e., rdf:seeAlso).

$\square$ Properties do not have uniform distribution in a dataset.

- Hence, some properties do not have enough matches or appearances.

- This is due to rare classes and domains they belong to.

- We can run the algorithm on instances that these less frequent properties appear iteratively.

$\square$ Current limitations,

- Requires ECR links

- Requires overlapping datasets

- Object-type properties

- Inability to identify property - sub property relationships 


\section{Roadmap}

Background

DStatistical Equivalence of properties

DEvaluation

$\square$ Discussion, interesting facts, and future directions

\section{$\square$ Conclusion}


$\square$ We approximate owl:equivalentProperty using Statistical Equivalence of properties by analyzing property extensions, which is schema independent.

$\square$ This novel extension based approach works well with interlinked datasets.

$\square$ The extension based approach outperforms syntax or dictionary based approaches. $\mathrm{F}$ measure gain in the range of $57 \%-78 \%$.

$\square$ It requires many comparisons, but can be easily parallelized evidenced by our Map-Reduce implementation. 


\section{Questions?}

Thank You

http://knoesis.wright.edu/researchers/kalpa kalpa@knoesis.org

Kno.e.sis - Ohio Center of Excellence in Knowledge-enabled Computing Wright State University, Dayton, Ohio, USA 